\title{
Shaping the dynamic mitochondrial network
}

\author{
Laura L Lackner
}

\begin{abstract}
In a majority of cell types, mitochondria form highly dynamic, tubular networks. Maintaining the shape of this complex network is critical for both mitochondrial and cellular function and involves the activities of mitochondrial division, fusion, motility, and tethering. Recent studies have advanced our understanding of the molecular mechanisms underlying these conserved activities and their integration with cellular needs.
\end{abstract}

Mitochondria are not discrete or autonomous but form highly dynamic, interconnected networks whose biogenesis and structure are highly influenced by the needs of the cell (Figure 1a,b). Mitochondria have a myriad of functions in addition to cellular energy production and play critical roles in cell cycle progression, differentiation, development, immune responses, lipid and calcium homeostasis, and apoptotic cell death (Figure 1c; reviewed in [1]). These diverse roles of mitochondria are intimately connected to the structure and cellular context of the essential organelle. Thus, it is not surprising that aberrant mitochondrial architecture has been associated with an ever-increasing number of diseases.

The shape and cellular distribution of the mitochondrial network is maintained in large part by the conserved activities of mitochondrial division, fusion, motility and tethering (Figure 2). These conserved activities are coordinately regulated and fully integrated with cellular physiology to respond to the rapidly changing needs of the cell. For example, mitochondria elongate during the G1/S transition, fragment at the onset of mitosis and apoptosis, hyperfuse in response to nutrient starvation and oxidative stress, and are recruited to and maintained at active synapses [2-8]. This regulated restructuring of mitochondria is functionally significant as disruption of these processes has negative effects on overall cellular function.

Correspondence: Laura.Lackner@northwestern.edu

Department of Molecular Biosciences, Northwestern University, 2205 Tech Drive Hogan 2-100, Evanston, IL 60208, USA
Great progress has been made in our understanding of the molecular mechanisms that actively shape the dynamic mitochondrial network. However, we still have much to learn regarding the coordinate regulation of the activities that drive the context-specific changes in mitochondrial form and function. This review will highlight recent advances in our understanding of the molecular mechanisms that impact mitochondrial form and the integration of these mechanisms with one another and with cellular function.

\section{Mitochondrial division and fusion: regulators of mitochondrial connectivity}

The antagonistic activities of mitochondrial division and fusion are required to maintain the form and function of mitochondria (Figure 2). Mitochondrial fusion facilitates communication and sharing of contents between mitochondrial compartments, which can buffer transient defects in mitochondrial function [9]. Mitochondrial division facilitates the transport, distribution, and quality control-mediated degradation of the organelle [10]. The dynamic processes of mitochondrial division and fusion are mediated by dynamin related proteins (DRPs). DRPs are a family of large GTPases that harness GTP-dependent self-assembly and subsequent GTP hydrolysis-mediated conformational changes to remodel membranes [11,12]. The DRP Dnm1/Drp1 (yeast/mammals) drives the scission of mitochondrial membranes, and the DRPs Fzo1/Mfn1/2 and Mgm1/Opa1 mediate fusion of the mitochondrial outer and inner membranes (MOM and MIM), respectively [13].

\section{Mitochondrial division}

The dynamin related GTPase Dnm1/Drp1 is a core component of the mitochondrial division machine (Figure 3a) [14-18]. Dnm1/Drp1 assembles into helical structures that wrap around mitochondria and mediate the scission of mitochondrial membranes [17,19-21]. GTP binding drives Dnm1/Drp1 helix assembly, which in turn triggers GTP hydrolysis via the formation of a catalytic interface between the GTPase domains of molecules in adjacent helical rungs [19,22-25]. Consequent GTP hydrolysis- 

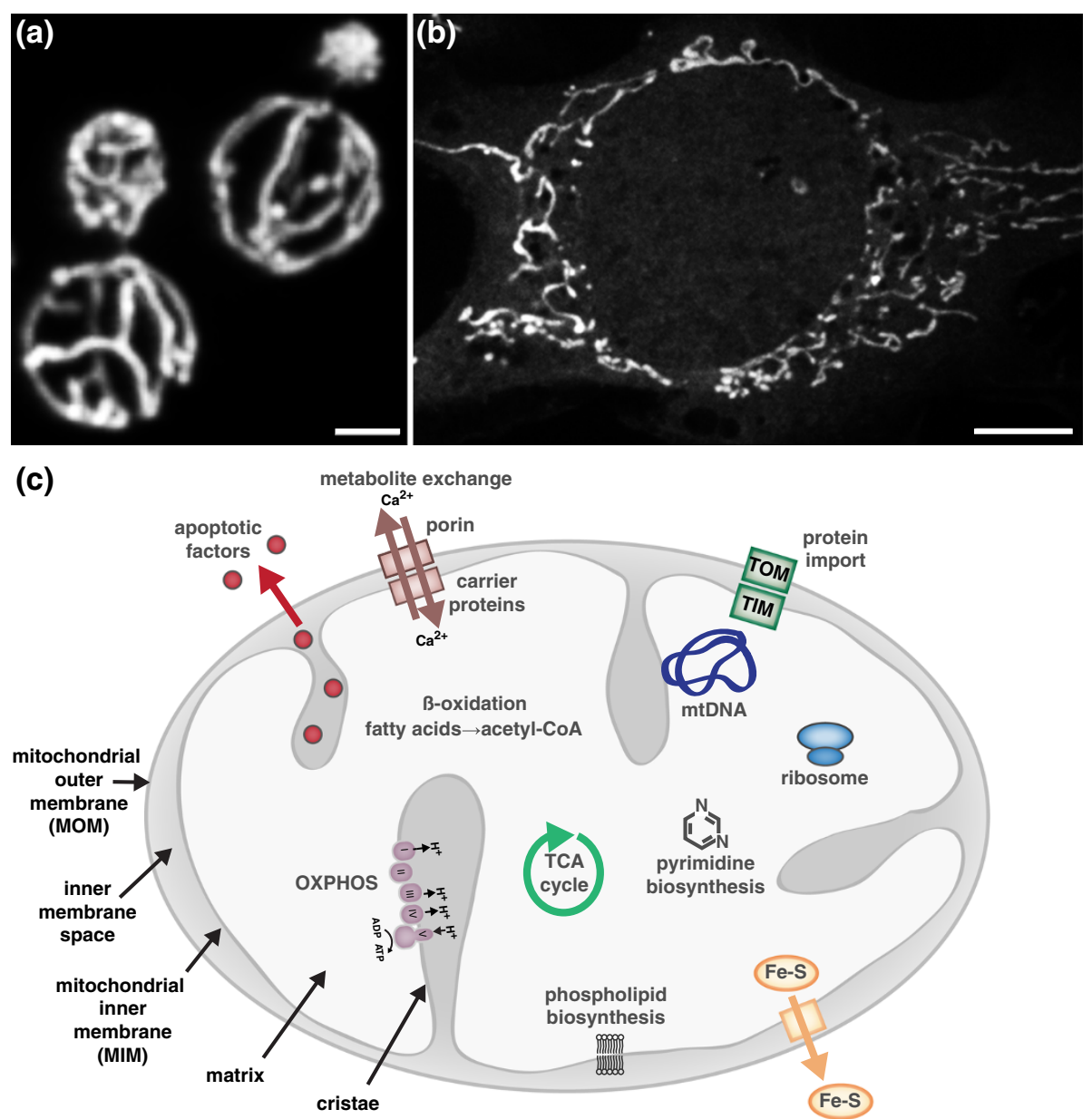

Figure 1. The form and functions of mitochondria. (a) In yeast, mitochondria form a connected, tubular network that is evenly distributed at the cell cortex. (b) Mitochondria also form well-distributed tubular networks in a majority of mammalian cell types. The mitochondrial network of a mouse embryonic fibroblast is shown. Scale bar, $2 \mu$ for (a,b). (c) Like their bacterial ancestors, mitochondria possess two structurally and functionally distinct membranes, the mitochondrial outer and inner membranes (MOM and MIM, respectively). The MOM and MIM surround two compartments, the inner membrane space and matrix, respectively. The matrix houses the circular mitochondrial genome (mtDNA), which encodes protein components of the respiratory complexes I to IV. The MIM, the most protein dense membrane in the cell, adopts elaborate folds called cristae in which assembled respiratory complexes are housed. In addition to ATP production via oxidative phosphorylation, mitochondria play critical roles in phospholipid biosynthesis, metabolite exchange/buffering, $\beta$-oxidation of fatty acids, iron-sulfur cluster biogenesis, pyrimidine biosynthesis and the storage and release of apoptotic factors (reviewed in [1]). TCA, tricarboxylic acid.

driven conformational changes in the helix result in further constriction and ultimate scission of the underlying mitochondrial membranes [26,27].

Drp1-mediated membrane remodeling is subject to regulation at various points in the division pathway, including targeting of Drp1 to the MOM and modulation of the GTP-regulated assembly, constriction, and disassembly of the Drp1 helix. Post-translational modification (PTM) of Drp1, which includes phosphorylation, sumoylation, ubiquitination, nitrosylation and O-glycosylation, can positively or negatively impact Drp1 activity (reviewed in [28]), and alternative RNA splicing produces functionally distinct Drp1 isoforms, which themselves are subject to differential PTM [29]. The activity of the mitochondrial division DRP is also regulated by protein effectors (adaptors): Mdv1 in yeast and Fis1, Mff, MiD49 and MiD51 in mammals [30-36]. These effectors function to target and/or regulate the assembly of the division DRP on the mitochondrial surface, providing critical spatial and temporal regulation [37-40].

Distinct combinations of Drp1 isoforms, effectors and PTMs provide contextual regulation to Drp1 activity and allow for the integration of mitochondrial division with cellular needs. For example, site-specific phosphorylation activates Drp1 activity during mitosis, which facilitates segregation of mitochondria into daughter cells [3]. During nutrient starvation, the phosphorylation of one site and dephosphorylation of another attenuate Drp1 activity, leading to mitochondrial elongation, which protects mitochondria from autophagic degradation and sustains 


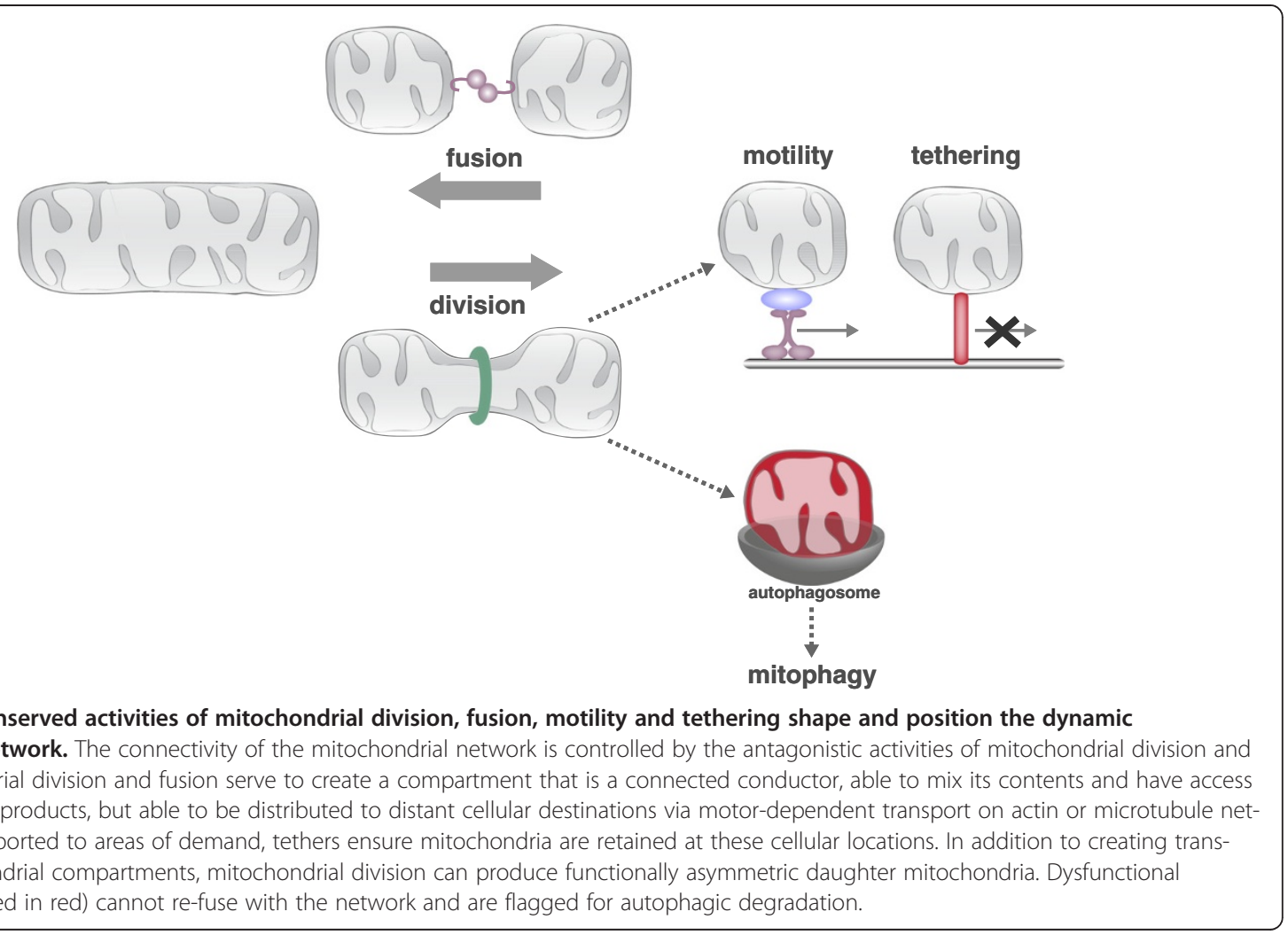

cell viability $[4,5]$. Phosphorylation has recently been shown to regulate the association of splice-specific isoforms of Drp1 with microtubules (MTs) [29]. MTassociated Drp1 is a latent form of Drp1 that can be selectively mobilized by cyclin-dependent kinase signaling, providing a novel mechanism to integrate Drp1 activity with the cell cycle. In addition, recent structural analyses of the Drp1 effector MiD51 identified ADP as an unexpected regulator of mitochondrial division [38,41]. MiD51 adopts a nucleotidyltransferase fold that can bind ADP. MiD51 mutants deficient in ADP binding are able to recruit Drp1 to mitochondria $[38,41]$ and support mitochondrial division under normal conditions [41]. However, stress-induced mitochondrial division is attenuated in the absence of ADP-binding [38], providing context-specific regulation to the activity of the division effector.

While protein and PTM effectors can function to target the mitochondrial division DRP to the mitochondrial surface, the amalgamation of recent studies indicates that mitochondrial division site selection involves communication between unexpected extramitochondrial factors and internal mitochondrial structures. The initial observation that the ER physically wraps around mitochondria and facilitates mitochondrial constriction at nascent division sites added a novel player to the mitochondrial division pathway [42]. ER-mediated mitochondrial constriction occurs upstream of Drp1 recruitment and represents an early stage in mitochondrial division $[21,42]$. In mammalian cells, an ER-associated formin, INF2, has been functionally linked to this early stage mitochondrial constriction [43]. Evidence indicates that INF2 mediates actin polymerization and subsequent myosin recruitment to sites of ER-mitochondria contact, providing a force-generating mechanism to drive the constriction of mitochondria [44]. This initial constriction likely generates a geometric hotspot that is more favorable for Drp1 helix assembly [42]. Evidence hints that actin may play a similar role in mitochondrial division in yeast. In yeast, the multi-subunit ER-mitochondria encounter structure (ERMES), which tethers the ER and mitochondria, is both spatially and functionally linked to sites of mitochondrial division [45]. The ERMES complex has also been implicated as a bridge between mitochondria and the actinomyosin network and thus may function at division sites to coordinate the recruitment of cytoskeletal and motor proteins [46].

The spatial and functional link between ERMES and mitochondrial division also place a matrix-localized factor, the mitochondrial nucleoid, at the site of mitochondrial division. The mitochondrial nucleoid is composed of mitochondrial DNA (mtDNA) and proteins required for its compaction and maintenance. A subset of mitochondrial nucleoids, specifically those that are actively replicating, co-localize with the ERMES complex [47]. 


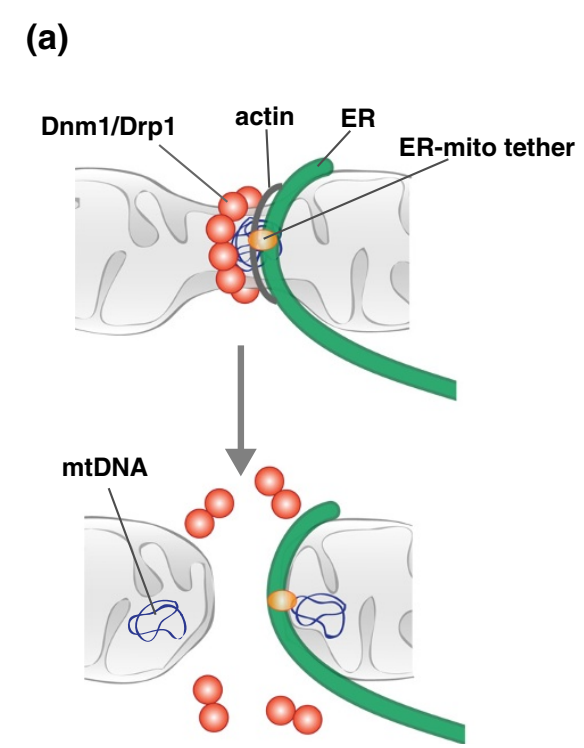

(b)

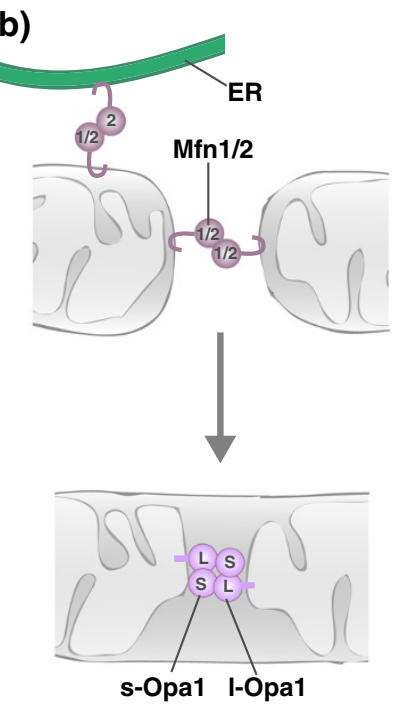

Figure 3. Molecular models of mitochondrial division and fusion. (a) Mitochondrial division involves communication between extramitochondrial division factors and internal mitochondrial structures. See text for details. (b) Mitochondrial fusion requires the sequential interaction of the MOM and MIM. MOM fusion is mediated by Mfn1/2, and MIM fusion is mediated by Opa1. Mfn2 is also localized to the ER and functions to tether the ER and mitochondria.

ERMES-associated nucleoids are present at a majority of mitochondrial division sites, and nucleoids are often found in both of the newly generated mitochondrial tips following division [45]. As tips can go on to fuse with other parts of the mitochondrial network, the placement of nucleoids in newly generated mitochondrial tips provides a means to redistribute mtDNA within the network. The spatial association of nucleoids with mitochondrial division sites is conserved, and defects in mitochondrial division lead to defects in nucleoid distribution in both yeast and mammalian cells $[48,49]$. While homologs of the core ERMES subunits are not found in higher eukaryotes, the putative ERMES regulatory subunit Gem1 (Miro 1 and 2 in humans) is conserved [50,51]. Whether Miro associates with and regulates a functionally analogous ERmitochondria tether in higher eukaryotes is an outstanding question. It is likely additional factors that mediate physical and functional interactions between matrix-localized mitochondrial nucleoids and the MOM-associated division machinery will be identified. Indeed, based on the recent addition of novel, unexpected players to the mitochondrial division pathway in both yeast and humans, it is clear that our knowledge of the entire complement of proteins that comprise and regulate the division complex is far from complete.

\section{Mitochondrial fusion}

In comparison to the division DRPs, less is known about the mechanism by which fusion DRPs harness GTPdriven self-assembly and GTP hydrolysis-mediated conformational changes to fuse membranes. Current evidence indicates that MOM and MIM fusion proceed via two separable stages, membrane tethering and lipid content mixing, both of which require fusion DRPs (Figure $3 \mathrm{~b}$ ) [52-58]. Membrane tethering is mediated by fusion DRP self-assembly, and subsequent GTP hydrolysis-induced conformational changes are proposed to destabilize the lipid bilayers of the tethered fusion partners to facilitate lipid mixing and fusion. While assembled structures of the fusion DRPs have been observed by electron microscopy, it is not yet clear if and how these structures correlate to active fusion complexes [59-62].

Like the mitochondrial division DRP, the mitochondrial fusion DRPs are subject to various levels of regulation, including alternative splicing, PTM, proteolytic processing, and regulated protein degradation, all of which can link mitochondrial fusion with cellular physiology. For example, one of the peptidases responsible for Opa1 processing, Omal, has been shown to be activated in response to various cellular stressors [63]. Under these conditions, enhanced Opa1 processing correlates with attenuated fusion and stress-induced mitochondrial fragmentation. In contrast, OXPHOS-stimulated processing of Opa1 via the peptidase Yme1L has been shown to stimulate MIM fusion [64]. Recent work demonstrates that acetylation can also regulate Opa1 activity. Acetylation of OPA1 reduces its activity, and the acetylated state and thus function of OPA1 can be modulated by the mitochondrial deacetylase Sirt3 [65]. As the sirtuin is dependent on $\mathrm{NAD}^{+}$, its activity is highly sensitive to the metabolic state of the cell. Thus, context-specific processing and PTM of Opa1 link MIM fusion to both cellular health and metabolism. Acetylation 
is also proposed to regulate MOM fusion under certain stress conditions by promoting the ubiquitination and subsequent degradation of Mfn1 [66]. Phosphorylation and ubiquitination can also trigger $\mathrm{Mfn} 1 / 2$ degradation and thus inhibit MOM fusion in response to specific stimuli [67-69]. In addition to promoting degradation, site-specific ubiquitination can stabilize MOM fusion DRPs, as has been shown for Fzo1, and promote fusion, perhaps via the stabilization of Fzo1 oligomers [70]. Regulation of the oligomeric state of Mfn2 via modification by oxidized glutathione has also been proposed to promote mitochondrial fusion [71]. Additionally, localization of Mfn2 to both the MOM and ER raises the possibility that differential targeting of the protein can also be used as a means to regulate fusion. As interactions between ER-associated Mfn2 and mitochondrial-associated Mfn1/2 function to tether the two organelles [72], ER-mitochondria contacts may also play direct and/or regulatory roles in mitochondrial fusion. Given the complex regulation of mitochondrial fusion at the level of both the MOM and MIM, and the likelihood that additional regulatory mechanisms will be identified, we have a challenging road ahead in the pursuit of a complete understanding of how mitochondrial fusion is fully integrated with cellular needs.

\section{The integration of mitochondrial division and fusion}

The relative rates of mitochondrial division and fusion modulate the connectivity of the mitochondrial network. Under normal conditions, the rates of mitochondrial division and fusion in yeast are balanced, suggesting that there is coordinate regulation of the two processes [73]. A reported interaction between Drp1 and Mfn2 raises the possibility that mitochondrial dynamics may be coordinated via direct interactions between the division and fusion machinery [74]. Further support of coordinate regulation comes from recent work that both spatially and functionally links the short form of Opa1 to mitochondrial division [75]. Non-processed forms of Opa1 (long Opa1) are sufficient to mediate mitochondrial fusion, while the accumulation of processed forms (short Opa1) correlate with mitochondrial fragmentation without negatively effecting fusion rates, indicative of a role for short Opa1 in division. Consistently, a catalytically inactive form of short Opa1 partially co-localizes with Drp1 and sites of ER-mitochondria contact. This functional and spatial association of short Opa1 with mitochondrial division suggests that the processing of Opa1 can function to balance mitochondrial division and fusion dynamics.

\section{Mitochondrial motility and tethering: regulators of mitochondrial position}

The activities of mitochondrial motility and tethering impact the overall cellular distribution of mitochondria.
These activities are critical to ensure mitochondria are trafficked to and maintained at the cellular locations where they are needed.

\section{Mitochondrial motility and tethering in yeast}

In the simple polarized budding yeast cell, mitochondrial motility and tethering are critical to ensure that the daughter cell inherits and mother cell retains the essential mitochondrial compartment (Figure 4a). Mitochondria are actively transported to the growing bud via Myo2-driven transport along the actin cytoskeleton [76-82]. While transport is critical to place mitochondria in the bud, mother- and bud-specific mitochondrial tethers ensure that both cells retain part of the essential mitochondrial network [83-85]. Interestingly, daughters are born with a constant mitochondrial content to cell size ratio, suggesting that there is communication between mitochondrial transport and biogenesis and cell growth pathways in yeast [86].

The yeast mitochondrial inheritance mechanism is also harnessed to contribute to mother-daughter age asymmetry, whereby daughter cells are born young despite the age of the mother [87]. In yeast, mitochondria retained in the mother cell have lower membrane and redox potential and higher superoxide levels than those inherited by the daughter [88]. How this asymmetry in mitochondrial function is achieved is not clear; however, selective transport and retention pathways likely play a role. Indeed, in the absence of the Myo2-mitochondrial adaptor/bud-specific tether Mmr1, mother-daughter age asymmetry is disrupted [88]. Whether the motherspecific tether also plays a role in the establishment of mother-daughter age asymmetry and how the selective transport and tethering of mitochondria are achieved are at this point unclear.

\section{Mitochondrial motility and tethering in neurons}

In more complex polarized cells such as neurons and immune cells, mitochondria must be actively transported to and maintained in active synaptic regions (Figure $4 \mathrm{~b}$ ), which have high demands for energy and $\mathrm{Ca}^{2+}$ buffering. Disrupting the synaptic translocation of mitochondria adversely affects both neuronal and immune cell function $[6-8,89]$. Due to the uniform, polarized arrangement of MTs in axons and the critical importance of mitochondrial positioning to neuronal function, neurons have proven to be an excellent model system to study regulated mitochondrial transport and tethering (for in depth review see $[90,91])$. In neurons, the kinesin Kif5 is the main motor driving anterograde mitochondrial transport, while the retrograde transport of mitochondria is driven by dynein. Adaptor proteins mediate the interactions between these cytoskeletal motors and mitochondria. The adaptor protein Milton/TRAK bridges the interaction 


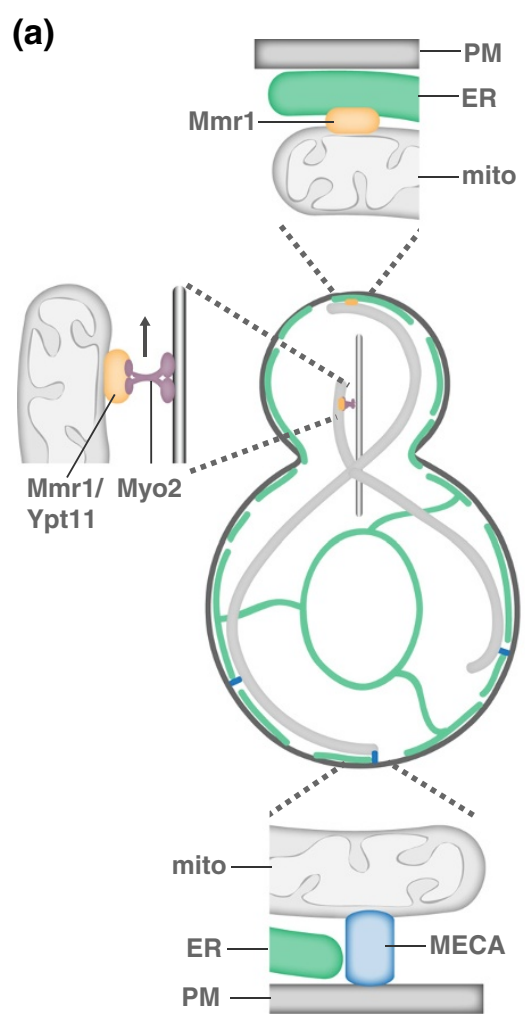

(b)
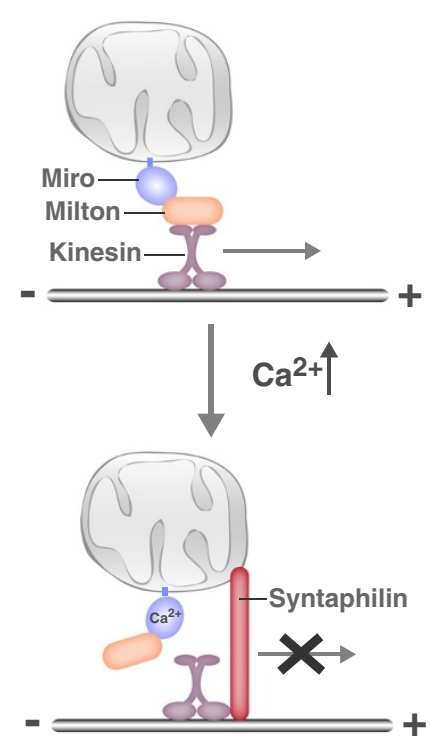

Figure 4. Molecular models of mitochondrial motility and tethering. (a) In yeast, mitochondria are actively transported to the growing bud via Myo2-driven transport along the actin cytoskeleton. Myo2-driven transport requires either Mmr1 or Ypt11 [76-82]. Mother- and bud-specific mitochondrial tethers ensure that both cells retain part of the essential mitochondrial compartment. The mother specific tether MECA (mitochondria-ER-cortex anchor) is composed of three membranes, the plasma membrane (PM), ER and mitochondria, and at least two proteins, Num1 and Mdm36 [84]. In addition to serving as a mitochondrial adaptor for Myo2, Mmr1 functions to tether mitochondria to ER sheets at the bud tip [83]. (b) A model for activity-dependent transport and tethering of mitochondria in axons. The conserved MOM Rho-like GTPase Miro and its binding partner Milton function as a mitochondrial receptor for kinesin. In active synaptic regions, $\mathrm{Ca}^{2+}$-binding by Miro triggers a confirmation change that disrupts kinesin-driven mitochondrial transport $[98,99]$. $\mathrm{Ca}^{2+}$-mediated confirmation changes have been proposed to disrupt the interaction between Miro/Milton with kinesin (shown here) or kinesin with microtubules. In response to neuronal activity (elevated $\mathrm{Ca}^{2+}$ ), syntaphilin is also recruited to mitochondria and functions as a static mitochondria-microtubule tether [100].

between Kif5 and the MOM receptor Miro, a Rho-like GTPase with two GTPase domains, two $\mathrm{Ca}^{2+}$-binding EF hand motifs and two recently identified hidden EF-hand motifs that bind a helix that structurally mimics an EF hand ligand [92-94]. Miro likely also serves as the mitochondrial receptor for dynein as both anterograde and retrograde mitochondrial transport are disrupted in the absence of Miro and Miro's binding partner TRAK interacts with both kinesin and dynein motors $[95,96]$.

Miro has been proposed to function in activity-dependent regulation of mitochondrial transport via its ability to sense calcium [97-99]. A relatively small proportion of mitochondria are motile in neurons, and this motile population is further decreased in response to synaptic activity, which increases local $\mathrm{Ca}^{2+}$ concentrations. $\mathrm{Ca}^{2+}$ binding by Miro is proposed to trigger conformational changes that disrupt the interaction between Kif5 and MTs or Milton/TRAK and Kif5 and, consequently, disrupt mitochondrial transport $[98,99]$. Interestingly, retrograde transport does not take over in this circumstance, suggesting that dyneindependent transport is also altered in response to elevated $\mathrm{Ca}^{2+}$ and/or a mitochondrial anchoring mechanism is activated. A recent study has elegantly dissected an activitydependent anchoring mechanism [100]. The activitydependent immobilization of axonal mitochondria requires the neuron-specific MOM protein syntaphilin [101]. In response to neuronal activity, syntaphilin is recruited to axonal mitochondria where it functions as an activityregulated brake to mitochondrial transport. Syntaphilin competes with Milton/TRAK for Kif5 binding, and once bound to Kif5, syntaphilin inhibits the activity of the motor protein [100]. What mediates the activity-dependent recruitment of syntaphilin to axonal mitochondria and how the syntaphilin-mediated brake is released are outstanding questions. Interestingly, syntaphilin is not required for the activity-dependent immobilization of mitochondria in dendrites, indicating that a dendrite-specific tethering mechanism exists [100]. Consistently, mitochondrial motility in 
dendrites is primarily dynein-driven, supporting the need for a kinesin-independent tethering mechanism [96]. With the exception of the axonal-specific tether syntaphilin, the same motor and adaptor proteins and regulatory mechanisms that govern neuronal mitochondrial transport appear to be employed for mitochondrial transport in nonneuronal cell types $[97,102]$.

\section{The role of the ER in mitochondrial positioning}

In yeast, the ER is a component of both the mother- and bud-specific mitochondrial tethers $[83,84]$. Whether the ER is a conserved component of positional mitochondrial tethers in other cell types remains to be determined. In activated $\mathrm{T}$ cells, both mitochondria and the ER are recruited to and maintained at the immune synapse $[8,103]$. Thus, ER-mitochondria tethers, such as Mfn2, may play a role in the synaptic translocation of mitochondria. Miro has also been localized to ER-mitochondria contacts in mammalian cells, raising the possibility that Miro-mediated ER-mitochondria tethering may also function in the positioning of mitochondria [50].

\section{Additional functions of mitochondrial tethers}

Mitochondrial tethers are not only important for positioning mitochondria relative to overall cellular structure but also play critical roles in positioning mitochondria relative to other organelles. The juxtaposition of membrane systems can facilitate the exchange of lipid, calcium and/or other small molecules between tethered compartments [104]. Indeed, the ER-mitochondria tether ERMES has been functionally linked to lipid transport between the ER and mitochondria in yeast [105], and ER-mitochondrial tethering by Mfn2 functions in $\mathrm{Ca}^{2+}$ signaling and lipid synthesis/transport between the two organelles [72,106]. In addition, Mfn2-mediated ERmitochondrial contact plays a role in autophagosome biogenesis [107], and an ER-mitochondria tether composed of the ER protein Bap31 and mitochondrial anchored Fis1 is functionally associated with apoptosis as well as the removal of defective mitochondria by mitophagy $[108,109]$. Thus, mitochondrial tethers mediate functional interactions between the ER and mitochondria that are critical for many key cellular homeostatic pathways.

In addition to physical and functional connections to the ER, a recent study demonstrates that mitochondria are physically and functionally tethered to melanosomes, specialized lysosome-related organelles of pigment cells [110]. Mitochondria-melanosome tethering is mediated by Mfn2 and plays a role in melanosome biogenesis. Mitochondria also have close physical association with lipid droplets, although the molecular basis and functional consequences of this association are not known [111]. Given the number of ER-mitochondria contacts predicted for a single yeast cell alone is approximately 100 [112], it is likely that many additional inter-organelle tethers will be identified, many of which may be functionally distinct.

\section{Further integration of mitochondrial dynamics, motility and tethering pathways}

Growing evidence suggests the activities of mitochondrial division, fusion, motility and tethering are interdependent and that the disruption of one activity can have indirect consequences on another. Indeed, attenuation of mitochondrial division disrupts the transport of mitochondria to neuronal and immune synapses, ultimately leading to detrimental effects on cellular function [6-8,89]. Mitochondrial tethering defects can reduce the rates of mitochondrial division [84,113,114], perhaps by disrupting the membrane tension required for DRPmediated membrane scission [115]. In addition, mitochondrial transport is disrupted in the absence of the MOM fusion DRP Mfn2 [116]. Therefore, in addition to understanding each dynamic attribute in isolation, we must carefully consider the complex relationships that exist between the activities themselves and with the rest of the cell.

\section{Acknowledgments}

I would like to thank Suzanne Hoppins and Holly Ping for helpful discussions and comments. I would like to apologize to colleagues whose outstanding work on mitochondrial division, fusion, motility and tethering could not be included due to space limitations.

\section{Published: 27 May 2014}

\section{References}

1. Nunnari J, Suomalainen A: Mitochondria: in sickness and in health. Cell 2012, 148:1145-1159.

2. Mitra K, Wunder C, Roysam B, Lin G, Lippincott-Schwartz J: A hyperfused mitochondrial state achieved at G1-S regulates cyclin E buildup and entry into S phase. Proc Natl Acad Sci U S A 2009, 106:11960-11965.

3. Taguchi N, Ishihara N, Jofuku A, Oka T, Mihara K: Mitotic phosphorylation of dynamin-related GTPase Drp1 participates in mitochondrial fission. J Biol Chem 2007, 282:11521-11529.

4. Gomes LC, Di Benedetto G, Scorrano L: During autophagy mitochondria elongate, are spared from degradation and sustain cell viability. Nat Cell Biol 2011, 13:589-598.

5. Rambold AS, Kostelecky B, Elia N, Lippincott-Schwartz J: Tubular network formation protects mitochondria from autophagosomal degradation during nutrient starvation. Proc Natl Acad Sci U S A 2011, 108:10190-10195.

6. Li Z, Okamoto K, Hayashi Y, Sheng M: The importance of dendritic mitochondria in the morphogenesis and plasticity of spines and synapses. Cell 2004, 119:873-887.

7. Verstreken P, Ly CV, Venken KJ, Koh TW, Zhou Y, Bellen HJ: Synaptic mitochondria are critical for mobilization of reserve pool vesicles at Drosophila neuromuscular junctions. Neuron 2005, 47:365-378.

8. Quintana A, Schwindling C, Wenning AS, Becherer U, Rettig J, Schwarz EC, Hoth M: T cell activation requires mitochondrial translocation to the immunological synapse. Proc Natl Acad Sci U S A 2007, 104:14418-14423.

9. Chen $\mathrm{H}$, Chan DC: Physiological functions of mitochondrial fusion. Ann N Y Acad Sci 2010, 1201:21-25.

10. Lackner LL, Nunnari JM: The molecular mechanism and cellular functions of mitochondrial division. Biochim Biophys Acta 2009, 1792:1138-1144. 
11. Faelber K, Gao S, Held M, Posor Y, Haucke V, Noe F, Daumke O: Oligomerization of dynamin superfamily proteins in health and disease. Prog Mol Biol Trans/ Sci 2013, 117:411-443.

12. Praefcke GJ, McMahon HT: The dynamin superfamily: universal membrane tubulation and fission molecules? Nat Rev Mol Cell Biol 2004, 5:133-147

13. Hoppins $S$, Lackner $L$, Nunnari J: The machines that divide and fuse mitochondria. Annu Rev Biochem 2007, 76:751-780.

14. Bleazard W, McCaffery JM, King EJ, Bale S, Mozdy A, Tieu Q, Nunnari J, Shaw JM: The dynamin-related GTPases, Dnm1, regulates mitochondrial fission in yeast. Nature Cell Biol 1999, 1:298-304.

15. Otsuga D, Keegan BR, Brisch E, Thantcher JW, Hermann GJ, Bleazard W, Shaw J: The dynamin GTPase, Dnm1p, controls mitochondrial morphology in yeast. J Cell Biol 1998, 143:333-349.

16. Sesaki $H$, Jensen RE: Division versus fusion: Dnm1p and Fzo1p antagonistically regulate mitochondrial shape. J Cell Biol 1999 147:699-706.

17. Smirnova E, Griparic L, Shurland DL, van Der Bliek AM: Dynamin-related protein drp1 is required for mitochondrial division in mammalian cells. Mol Biol Cell 2001, 12:2245-2256.

18. Smirnova E, Shurland D, Ryazantsev S, Van Der Bliek A: A human dynamin-related protein controls the distribution of mitochondria. J Cell Biol 1998, 143:351-358.

19. Ingerman E, Perkins EM, Marino M, Mears JA, McCaffery JM, Hinshaw JE, Nunnari J: Dnm1 forms spirals that are structurally tailored to fit mitochondria. J Cell Biol 2005, 170:1021-1027.

20. Labrousse AM, Zappaterra MD, Rube DA, van der Bliek AM: C. elegans dynamin-related protein DRP-1 controls severing of the mitochondrial outer membrane. Mol Cell 1999, 4:815-826.

21. Legesse-Miller A, Massol RH, Kirchhausen T: Constriction and dnm1p recruitment are distinct processes in mitochondrial fission. Mol Biol Cell 2003, 14:1953-1963.

22. Chappie JS, Acharya S, Leonard M, Schmid SL, Dyda F: G domain dimerization controls dynamin's assembly-stimulated GTPase activity. Nature 2010, 465:435-440.

23. Faelber K, Posor Y, Gao S, Held M, Roske Y, Schulze D, Haucke V, Noe F, Daumke O: Crystal structure of nucleotide-free dynamin. Nature 2011, 477:556-560.

24. Ford MG, Jenni S, Nunnari J: The crystal structure of dynamin. Nature 2011, 477:561-566.

25. Frohlich C, Grabiger S, Schwefel D, Faelber K, Rosenbaum E, Mears J, Rocks O, Daumke O: Structural insights into oligomerization and mitochondrial remodelling of dynamin 1-like protein. EMBO J 2013, 32:1280-1292.

26. Naylor K, Ingerman E, Okreglak V, Marino M, Hinshaw JE, Nunnari J: Mdv1 interacts with assembled $\mathrm{dnm} 1$ to promote mitochondrial division. J Biol Chem 2006, 281:2177-2183.

27. Mears JA, Lackner LL, Fang S, Ingerman E, Nunnari J, Hinshaw JE: Conformational changes in Dnm1 support a contractile mechanism for mitochondrial fission. Nat Struct Mol Biol 2011, 18:20-26.

28. Otera $\mathrm{H}$, Ishihara N, Mihara K: New insights into the function and regulation of mitochondrial fission. Biochim Biophys Acta 1833, 2013:1256-1268.

29. Strack S, Wilson TJ, Cribbs JT: Cyclin-dependent kinases regulate splice-specific targeting of dynamin-related protein 1 to microtubules. J Cell Biol 2013, 201:1037-1051.

30. Tieu $Q$, Nunnari J: Mdv1p is a WD repeat protein that interacts with the dynamin-related GTPase, Dnm1p, to trigger mitochondrial division. J Cell Biol 2000, 151:353-365

31. Cerveny KL, McCaffery JM, Jensen RE: Division of mitochondria requires a novel DNM1-interacting protein, Net2p. Mol Biol Cell 2001, 12:309-321.

32. Fekkes $P$, Shepard KA, Yaffe MP: Gag3p, an outer membrane protein required for fission of mitochondrial tubules. J Cell Biol 2000, 151:333-340.

33. Gandre-Babbe S, van der Bliek AM: The novel tail-anchored membrane protein Mff controls mitochondrial and peroxisomal fission in mammalian cells. Mol Biol Cell 2008, 19:2402-2412.

34. Otera H, Wang C, Cleland MM, Setoguchi K, Yokota S, Youle RJ, Mihara K. Mff is an essential factor for mitochondrial recruitment of Drp1 during mitochondrial fission in mammalian cells. J Cell Biol 2010, 191:1141-1158.

35. Palmer CS, Osellame LD, Laine D, Koutsopoulos OS, Frazier AE, Ryan MT: MiD49 and MiD51, new components of the mitochondrial fission machinery. EMBO Rep 2011, 12:565-573.

36. Zhao J, Liu T, Jin S, Wang X, Qu M, Uhlen P, Tomilin N, Shupliakov O, Lendahl U, Nister M: Human MIEF1 recruits Drp1 to mitochondrial outer membranes and promotes mitochondrial fusion rather than fission. EMBO J 2011, 30:2762-2778.

37. Lackner LL, Horner JS, Nunnari J: Mechanistic analysis of a dynamin effector. Science 2009, 325:874-877.

38. Loson OC, Liu R, Rome ME, Meng S, Kaiser JT, Shan SO, Chan DC: The Mitochondrial Fission Receptor MiD51 Requires ADP as a Cofactor. Structure 2014, 22:367-377.

39. Loson OC, Song Z, Chen H, Chan DC: Fis1, Mff, MiD49 and MiD51 mediate Drp1 recruitment in mitochondrial fission. Mol Biol Cell 2013, 24:659-667.

40. Koirala S, Guo Q, Kalia R, Bui HT, Eckert DM, Frost A, Shaw JM: Interchangeable adaptors regulate mitochondrial dynamin assembly for membrane scission. Proc Natl Acad Sci U S A 2013, 110:E1342-E1351.

41. Richter V, Palmer CS, Osellame LD, Singh AP, Elgass K, Stroud DA, Sesaki H, Kvansakul M, Ryan MT: Structural and functional analysis of MiD51, a dynamin receptor required for mitochondrial fission. J Cell Biol 2014 204:477-486.

42. Friedman JR, Lackner LL, West M, Dibenedetto JR, Nunnari J, Voeltz GK: ER Tubules Mark Sites of Mitochondrial Division. Science 2011, 334:358-362.

43. Korobova F, Ramabhadran V, Higgs HN: An actin-dependent step in mitochondrial fission mediated by the ER-associated formin INF2. Science 2013, 339:464-467.

44. Korobova F, Gauvin TJ, Higgs HN: A role for myosin II in mammalian mitochondrial fission. Curr Biol 2014, 24:409-414.

45. Murley A, Lackner LL, Osman C, West M, Voeltz GK, Walter P, Nunnari J: ER-associated mitochondrial division links the distribution of mitochondria and mitochondrial DNA in yeast. Elife 2013, 2:e00422.

46. Boldogh IR, Nowakowski DW, Yang HC, Chung H, Karmon S, Royes P, Pon $L A$ : A protein complex containing Mdm10p, Mdm12p, and Mmm1p links mitochondrial membranes and DNA to the cytoskeleton-based segregation machinery. Mol Biol Cell 2003, 14:4618-4627.

47. Meeusen S, Nunnari J: Evidence for a two membrane-spanning autonomous mitochondrial DNA replisome. J Cell Biol 2003, 163:503-510.

48. Ban-Ishihara R, Ishihara T, Sasaki N, Mihara K, Ishihara N: Dynamics of nucleoid structure regulated by mitochondrial fission contributes to cristae reformation and release of cytochrome c. Proc Natl Acad Sci U S A 2013, 110:11863-11868.

49. Itoh K, Tamura Y, lijima M, Sesaki H: Effects of Fcj1-Mos1 and mitochondrial division on aggregation of mitochondrial DNA nucleoids and organelle morphology. Mol Biol Cell 2013, 24:1842-1851.

50. Kornmann B, Osman C, Walter P: The conserved GTPase Gem1 regulates endoplasmic reticulum-mitochondria connections. Proc Natl Acad Sci US A 2011, 108:14151-14156.

51. Stroud DA, Oeljeklaus S, Wiese S, Bohnert M, Lewandrowski U, Sickmann A Guiard B, van der Laan M, Warscheid B, Wiedemann N: Composition and topology of the endoplasmic reticulum-mitochondria encounter structure. J Mol Biol 2011, 413:743-750.

52. Hermann GJ, Thatcher JW, Mills JP, Hales KG, Fuller MT, Nunnari J, Shaw JM: Mitochondrial fusion in yeast requires the transmembrane GTPase Fzo1p. J Cell Biol 1998, 143:359-374.

53. Santel A, Fuller MT: Control of mitochondrial morphology by a human mitofusin. J Cell Sci 2001, 114:867-874.

54. Chen H, Detmer SA, Ewald AJ, Griffin EE, Fraser SE, Chan DC: Mitofusins Mfn1 and Mfn2 coordinately regulate mitochondrial fusion and are essential for embryonic development. J Cell Biol 2003, 160:189-200.

55. Cipolat S, Martins de Brito O, Dal Zilio B, Scorrano L: OPA1 requires mitofusin 1 to promote mitochondrial fusion. Proc Natl Acad Sci U S A 2004, 101:15927-15932.

56. Meeusen S, McCaffery JM, Nunnari J: Mitochondrial fusion intermediates revealed in vitro. Science 2004, 305:1747-1752.

57. Meeusen S, Devay R, Block J, Cassidy-Stone A, Wayson S, McCaffery JM Nunnari J: Mitochondrial inner-membrane fusion and crista maintenance requires the dynamin-related GTPase Mgm1. Cell 2006, 127:383-395.

58. Song Z, Ghochani M, McCaffery JM, Frey TG, Chan DC: Mitofusins and OPA1 mediate sequential steps in mitochondrial membrane fusion. Mol Biol Cell 2009, 20:3525-3532.

59. DeVay RM, Dominguez-Ramirez L, Lackner LL, Hoppins S, Stahlberg H, Nunnari J: Coassembly of Mgm1 isoforms requires cardiolipin and mediates mitochondrial inner membrane fusion. J Cell Biol 2009, 186:793-803.

60. Rujiviphat J, Meglei G, Rubinstein JL, McQuibban GA: Phospholipid association is essential for dynamin-related protein Mgm1 to function in mitochondrial membrane fusion. J Biol Chem 2009, 284:28682-28686. 
61. Ban T, Heymann JA, Song Z, Hinshaw JE, Chan DC: OPA1 disease alleles causing dominant optic atrophy have defects in cardiolipin-stimulated GTP hydrolysis and membrane tubulation. Hum Mol Genet 2010, 19:2113-2122.

62. Abutbul-Ionita I, Rujiviphat J, Nir I, McQuibban GA, Danino D: Membrane tethering and nucleotide-dependent conformational changes drive mitochondrial genome maintenance (Mgm1) protein-mediated membrane fusion. J Biol Chem 2012, 287:36634-36638.

63. Baker MJ, Lampe PA, Stojanovski D, Korwitz A, Anand R, Tatsuta T, Langer T: Stress-induced OMA1 activation and autocatalytic turnover regulate OPA1-dependent mitochondrial dynamics. EMBO J 2014, 33:578-593.

64. Mishra P, Carelli V, Manfredi G, Chan DC: Proteolytic cleavage of opa1 stimulates mitochondrial inner membrane fusion and couples fusion to oxidative phosphorylation. Cell Metab 2014, 19:630-641.

65. Samant SA, Zhang HJ, Hong Z, Pillai VB, Sundaresan NR, Wolfgeher D, Archer SL, Chan DC, Gupta MP: SIRT3 deacetylates and activates OPA1 to regulate mitochondrial dynamics during stress. Mol Cell Biol 2014, 34:807-819.

66. Park YY, Nguyen OT, Kang H, Cho H: MARCH5-mediated quality control on acetylated Mfn1 facilitates mitochondrial homeostasis and cell survival. Cell Death Dis 2014, 5:e1172.

67. Gegg ME, Cooper JM, Chau KY, Rojo M, Schapira AH, Taanman JW: Mitofusin 1 and mitofusin 2 are ubiquitinated in a PINK1/parkin-dependent manner upon induction of mitophagy. Hum Mol Genet 2010, 19:4861-4870.

68. Poole AC, Thomas RE, Yu S, Vincow ES, Pallanck L: The mitochondrial fusion-promoting factor mitofusin is a substrate of the PINK1/parkin pathway. PLoS One 2010, 5:e10054.

69. Leboucher GP, Tsai YC, Yang M, Shaw KC, Zhou M, Veenstra TD, Glickman $\mathrm{MH}$, Weissman AM: Stress-induced phosphorylation and proteasomal degradation of mitofusin 2 facilitates mitochondrial fragmentation and apoptosis. Mol Cell 2012, 47:547-557.

70. Anton F, Dittmar G, Langer T, Escobar-Henriques M: Two deubiquitylases act on mitofusin and regulate mitochondrial fusion along independent pathways. Mol Cell 2013, 49:487-498.

71. Shutt T, Geoffrion M, Milne R, McBride HM: The intracellular redox state is a core determinant of mitochondrial fusion. EMBO Rep 2012, 13:909-915.

72. de Brito OM, Scorrano L: Mitofusin 2 tethers endoplasmic reticulum to mitochondria. Nature 2008, 456:605-610.

73. Nunnari J, Marshall W, Straight A, Murray A, Sedat JW, Walter P: Mitochondrial transmission during mating in S. cerevisiae is determined by mitochondrial fusion and fission and the intramitochondrial segregation of mtDNA. Mol Biol Cell 1997, 8:1233-1242.

74. Huang P, Galloway CA, Yoon Y: Control of mitochondrial morphology through differential interactions of mitochondrial fusion and fission proteins. PLOS One 2011, 6:e20655.

75. Anand R, Wai T, Baker MJ, Kladt N, Schauss AC, Rugarli E, Langer T: The i-AAA protease YME1L and OMA1 cleave OPA1 to balance mitochondrial fusion and fission. J Cell Biol 2014, 204:919-929.

76. Itoh T, Toh EA, Matsui Y: Mmr1p is a mitochondrial factor for Myo2p-dependent inheritance of mitochondria in the budding yeast. EMBO J 2004, 23:2520-2530.

77. Itoh T, Watabe A, Toh EA, Matsui Y: Complex formation with Ypt11p, a rab-type small GTPase, is essential to facilitate the function of Myo2p, a class $\mathrm{V}$ myosin, in mitochondrial distribution in Saccharomyces cerevisiae. Mol Cell Biol 2002, 22:7744-7757.

78. Altmann K, Frank M, Neumann D, Jakobs S, Westermann B: The class V myosin motor protein, Myo2, plays a major role in mitochondrial motility in Saccharomyces cerevisiae. J Cell Biol 2008, 181:119-130.

79. Eves PT, Jin Y, Brunner M, Weisman LS: Overlap of cargo binding sites on myosin V coordinates the inheritance of diverse cargoes. J Cell Biol 2012, 198:69-85.

80. Fortsch J, Hummel E, Krist M, Westermann B: The myosin-related motor protein Myo2 is an essential mediator of bud-directed mitochondrial movement in yeast. J Cell Biol 2011, 194:473-488.

81. Chernyakov I, Santiago-Tirado F, Bretscher A: Active segregation of yeast mitochondria by Myo2 is essential and mediated by Mmr1 and Ypt11. Curr Biol 2013, 23:1818-1824.

82. Lewandowska A, Macfarlane J, Shaw JM: Mitochondrial association, protein phosphorylation, and degradation regulate the availability of the active Rab GTPase Ypt11 for mitochondrial inheritance. Mol Biol Cell 2013, 24:1185-1195.

83. Swayne TC, Zhou C, Boldogh IR, Charalel JK, McFaline-Figueroa JR, Thoms S, Yang C, Leung G, Mclnnes J, Erdmann R, Pon LA: Role for cER and Mmr1p in anchorage of mitochondria at sites of polarized surface growth in budding yeast. Curr Biol 2011, 21:1994-1999.

84. Lackner LL, Ping H, Graef M, Murley A, Nunnari J: Endoplasmic reticulum-associated mitochondria-cortex tether functions in the distribution and inheritance of mitochondria. Proc Natl Acad Sci U S A 2013, 110:E458-E467.

85. Klecker T, Scholz D, Fortsch J, Westermann B: The yeast cell cortical protein Num1 integrates mitochondrial dynamics into cellular architecture. J Cell Sci 2013, 126:2924-2930.

86. Rafelski SM, Viana MP, Zhang Y, Chan YH, Thorn KS, Yam P, Fung JC, Li H, Costa Lda F, Marshall WF: Mitochondrial network size scaling in budding yeast. Science 2012, 338:822-824

87. Longo VD, Shadel GS, Kaeberlein M, Kennedy B: Replicative and chronological aging in Saccharomyces cerevisiae. Cell Metab 2012, 16:18-31.

88. McFaline-Figueroa JR, Vevea J, Swayne TC, Zhou C, Liu C, Leung G, Boldogh $I R$, Pon LA: Mitochondrial quality control during inheritance is associated with lifespan and mother-daughter age asymmetry in budding yeast. Aging Cell 2011, 10:885-895.

89. Baixauli F, Martin-Cofreces NB, Morlino G, Carrasco YR, Calabia-Linares C, Veiga E, Serrador JM, Sanchez-Madrid F: The mitochondrial fission factor dynamin-related protein 1 modulates T-cell receptor signalling at the immune synapse. EMBO J 2011, 30:1238-1250.

90. Schwarz TL: Mitochondrial trafficking in neurons. Cold Spring Harb Perspect Biol 2013, 5:a011304.

91. Sheng $\mathrm{ZH}$ : Mitochondrial trafficking and anchoring in neurons: New insight and implications. J Cell Biol 2014, 204:1087-1098.

92. Glater EE, Megeath LJ, Stowers RS, Schwarz TL: Axonal transport of mitochondria requires milton to recruit kinesin heavy chain and is light chain independent. J Cell Biol 2006, 173:545-557.

93. Fransson S, Ruusala A, Aspenstrom P: The atypical Rho GTPases Miro-1 and Miro-2 have essential roles in mitochondrial trafficking. Biochem Biophys Res Commun 2006, 344:500-510

94. Klosowiak JL, Focia PJ, Chakravarthy S, Landahl EC, Freymann DM, Rice SE: Structural coupling of the EF hand and C-terminal GTPase domains in the mitochondrial protein Miro. EMBO Rep 2013, 14:968-974.

95. Russo GJ, Louie K, Wellington A, Macleod GT, Hu F, Panchumarthi S, Zinsmaier KE: Drosophila Miro is required for both anterograde and retrograde axonal mitochondrial transport. J Neurosci 2009, 29:5443-5455.

96. van Spronsen M, Mikhaylova M, Lipka J, Schlager MA, van den Heuvel DJ, Kuijpers M, Wulf PS, Keijzer N, Demmers J, Kapitein LC, Jaarsma D, Gerritsen HC, Akhmanova A, Hoogenraad CC: TRAK/Milton motor-adaptor proteins steer mitochondrial trafficking to axons and dendrites. Neuron 2013, 77:485-502.

97. Saotome M, Safiulina D, Szabadkai G, Das S, Fransson A, Aspenstrom P, Rizzuto R, Hajnoczky G: Bidirectional Ca2 + -dependent control of mitochondrial dynamics by the Miro GTPase. Proc Natl Acad Sci U S A 2008, 105:20728-20733.

98. Macaskill AF, Rinholm JE, Twelvetrees AE, Arancibia-Carcamo IL, Muir J, Fransson A, Aspenstrom P, Attwell D, Kittler JT: Miro1 is a calcium sensor for glutamate receptor-dependent localization of mitochondria at synapses. Neuron 2009, 61:541-555.

99. Wang $X$, Schwarz TL: The mechanism of $\mathrm{Ca} 2+-$ dependent regulation of kinesin-mediated mitochondrial motility. Cell 2009, 136:163-174.

100. Chen Y, Sheng ZH: Kinesin-1-syntaphilin coupling mediates activitydependent regulation of axonal mitochondrial transport. J Cell Biol 2013, 202:351-364.

101. Kang JS, Tian JH, Pan PY, Zald P, Li C, Deng C, Sheng ZH: Docking of axonal mitochondria by syntaphilin controls their mobility and affects short-term facilitation. Cell 2008, 132:137-148.

102. Morlino G, Barreiro O, Baixauli F, Robles-Valero J, González-Granado JM, Villa-Bellosta R, Cuenca J, Sánchez-Sorzano CO, Veiga E, Martín-Cófreces NB, Sánchez-Madrid F: Miro-1 links mitochondria and microtubule Dynein motors to control lymphocyte migration and polarity. Mol Cell Biol 2014, 34:1412-1426.

103. Quintana A, Hoth M: Mitochondrial dynamics and their impact on T cell function. Cell Calcium 2012, 52:57-63.

104. Toulmay A, Prinz WA: Lipid transfer and signaling at organelle contact sites: the tip of the iceberg. Curr Opin Cell Biol 2011, 23:458-463.

105. Kornmann B, Currie E, Collins SR, Schuldiner M, Nunnari J, Weissman JS, Walter P: An ER-mitochondria tethering complex revealed by a synthetic biology screen. Science 2009, 325:477-481. 
106. Area-Gomez E, Del Carmen Lara Castillo M, Tambini MD, Guardia-Laguarta C, de Groof AJ, Madra M, Ikenouchi J, Umeda M, Bird TD, Sturley SL, Schon EA: Upregulated function of mitochondria-associated ER membranes in Alzheimer disease. EMBO J 2012, 31:4106-4123.

107. Hamasaki M, Furuta N, Matsuda A, Nezu A, Yamamoto A, Fujita N, Oomori H, Noda T, Haraguchi T, Hiraoka Y, Amano A, Yoshimori T: Autophagosomes form at ER-mitochondria contact sites. Nature 2013, 495:389-393.

108. Head BP, Zulaika M, Ryazantsev S, van der Bliek AM: A novel mitochondrial outer membrane protein, MOMA-1, that affects cristae morphology in Caenorhabditis elegans. Mol Biol Cell 2014, 22:831-841.

109. Iwasawa R, Mahul-Mellier AL, Datler C, Pazarentzos E, Grimm S: Fis1 and Bap31 bridge the mitochondria-ER interface to establish a platform for apoptosis induction. EMBO J 2011, 30:556-568.

110. Daniele T, Hurbain I, Vago R, Casari G, Raposo G, Tacchetti C, Schiaffino MV Mitochondria and melanosomes establish physical contacts modulated by Mfn2 and involved in organelle biogenesis. Curr Biol 2014, 24:393-403.

111. Zehmer JK, Huang Y, Peng G, Pu J, Anderson RG, Liu P: A role for lipid droplets in inter-membrane lipid traffic. Proteomics 2009, 9:914-921.

112. Achleitner G, Gaigg B, Krasser A, Kainersdorfer E, Kohlwein SD, Perktold A, Zellnig G, Daum G: Association between the endoplasmic reticulum and mitochondria of yeast facilitates interorganelle transport of phospholipids through membrane contact. Eur J Biochem 1999, 264:545-553.

113. Cerveny KL, Studer SL, Jensen RE, Sesaki H: Yeast mitochondrial division and distribution require the cortical num1 protein. Dev Cell 2007, 12:363-375

114. Hammermeister M, Schodel K, Westermann B: Mdm36 is a mitochondrial fission-promoting protein in Saccharomyces cerevisiae. Mol Biol Cell 2010, 21:2443-2452

115. Roux A, Uyhazi K, Frost A, De Camilli P: GTP-dependent twisting of dynamin implicates constriction and tension in membrane fission. Nature 2006, 441:528-531.

116. Misko A, Jiang S, Wegorzewska I, Milbrandt J, Baloh RH: Mitofusin 2 is necessary for transport of axonal mitochondria and interacts with the Miro/Milton complex. J Neurosci 2010, 30:4232-4240.

doi:10.1186/1741-7007-12-35

Cite this article as: Lackner LL: Shaping the dynamic mitochondrial network. BMC Biology 2014 12:35. 